\title{
Autonomous and Central Control of Production Networks
}

\author{
Sergey Dashkovskiy, Andrii Mironchenko and Lars Naujok
}

\begin{abstract}
In this contribution we discuss central and autonomous control of production networks. We provide a number of parameters that influence the comparative effectiveness of these two methods. Further, the results concerning stability of the production networks are discussed and it is shown, how the central planning methods, implemented into mathematical models with the help of optimal control theory, can be used to achieve information about the possible form of controls. We investigate the limitations of both control methods and give possible ways, how to increase the effectiveness of control of production networks.
\end{abstract}

\section{Authors profiles}

Sergey Dashkovskiy received the M.Sc. degree in applied mathematics and mechanics from the Lomonosov Moscow State University, Moscow, Russia in 1996 and the Ph.D. degree in mathematics from the University of Jena, Jena, Germany, in 2002. He is Head of the research group Mathematical Modeling of Complex Systems at the Center of Industrial Mathematics, University of Bremen, Germany. His research interests are in the field of nonlinear control theory and partial differential equations.

Andrii Mironchenko received the M.Sc degree in applied mathematics from Mechnikov Odessa National University in 2008. Since 2009 he is a research assistant in the Center for Industrial Mathematics at the University of Bremen. His research interests include stability of control systems, partial differential equations and their applications to logistics and biology.

Lars Naujok studied mathematics at the University of Bremen and received his diploma in 2008. Mr. Naujok is a research assistant in the Center for Industrial Mathematics at the University of Bremen since 2009. His research interests include stability and control of dynamical systems, Lyapunov methods, observers, fault detection and their applications to logistics. 


\section{Introduction}

Production networks are typical examples of large-scale and complex systems with a nonlinear behavior. By an increasing number of entities and material within the network the control of the material flows and the production rates of entities or machines is not an easy task. One way to achieve logistic goals, such as a high performance, robustness and stability, is the shift from centralized to decentralized or autonomous control.

The term production network is used to describe company or cross-company owned networks with geographically dispersed plants. These types of networks may react quickly on perturbations due to redundancies of common resources. But high flexibility causes interdependencies between production processes in different plants, e.g., allocation problems for products or planning of transports and transport capacity $[16,3]$. Therefore production planning and control (PPC) of production networks has to cover these tasks and also has to provide methods for an integrated planning and synchronization within the network, including planning of sales and inventory [28]. Under highly dynamic and complex conditions current PPC methods cannot cope with disturbances or unforeseen events in an appropriate manner [15]. Changing market conditions and inappropriate planning may cause uncertainties of lead times, inconstancy of schedules or may also lead to instability or even chaos.

The main idea of autonomous cooperating logistic processes is to enable intelligent logistic objects to route themselves through a logistic network according to their own objectives and to make and execute decisions, based on local information $[29,30]$. In this context intelligent logistic objects may be physical or material objects, e.g., parts or machines, as well as nonmaterial objects (e.g., production orders, information). It has been already shown that different autonomous control methods can help to increase the logistics performance and robustness of single production systems $[18,19,20]$. On the other hand, autonomously controlled production networks may show a sudden change of the dynamical systems behavior in dependence of varying start parameters and the logistic performance collapses in the sense of unpredictable and increasing throughput times and growing inventory [21]. Thus, investigations of the stability of autonomously controlled production networks are essential to understand the dynamical systems behavior.

Typical examples of unstable behavior are unbounded growth of unsatisfied orders or unbounded growth of the queue of the workload to be processed by a machine. This causes high inventory costs and loss of customers. To avoid instability of a network it is worth to investigate its behavior in advance. In particular, mathematical modeling and analysis provide helpful tools for design, optimization and control of such networks and for deeper understanding of their dynamical properties.

Roughly speaking, for production networks stability means that the state of the network remains bounded over time under bounded external inputs. In this contribution we identify the state as the number of unprocessed parts, which is the sum of the queue length and the work in progress (WIP). To identify parameters which guarantee stability of the network we are going to apply tools from mathematical systems 
theory. In this context mathematical models describing production network's behavior are needed.

In this contribution we consider the recent results in modeling of logistic networks, based on the input-to-state stability concepts, and analyze, in what form the limitations of control methods appear in the mathematical models of logistic processes. We argue, that in case, when the extensive information about the system is available, the central control may be quite effective, and can provide at least some important information about the structure of controls. On the other hand, if there is only a little information about the network, the autonomous control is a good alternative, but it rises the question about the stability of the network, which can be answered with the help of mathematical stability theory. We discuss these results from the viewpoint of limitations of autonomous control, and provide a number of parameters, that can be used to find the bounds, within which autonomous control is more effective, than central planning.

The structure of the contribution is as follows. In Section 2.1 we model general production network, which we analyze by means of optimal control methods in Section 2.2. In Section 2.3 we review recent results in autonomous control of production networks. Then we identify parameters of the network which influence the comparative effectiveness of different control methods in Subsection 2.4. The notions of stability and the tools to check whether a system is stable are presented in Section 3. In Section 4 we identify and discuss the limitations of autonomous control. Finally, the conclusions are given in Section 5.

Throughout the paper by $x^{T}$ we denote the transposition of a vector $x \in \mathbb{R}^{n}, n \in \mathbb{N}$ and $\mathbb{R}_{+}^{n}$ denotes the positive orthant $\left\{x \in \mathbb{R}^{n}: x \geq 0\right\}$ where we use the standard partial order for $x, y \in \mathbb{R}^{n}$ given by

$$
x \geq y \Leftrightarrow x_{i} \geq y_{i}, i=1, \ldots, n \text { and } x \geq y \Leftrightarrow \exists i: x_{i}<y_{i} .
$$

\section{Modeling and control of production networks}

\subsection{Description and Modeling of a general production network}

We are going to construct a model of a production network, consisting of $n$ market entities ( $n$ is an arbitrary positive number), which may be, for example, raw material suppliers (e.g., extracting or agricultural companies), producers, distributors and consumers. Each entity is a subsystem of the whole network. For simplicity we assume, that there is only one unified type of material, i.e., all primary products, used in the production network, can be measured as a number of units of this unified material.

We assume, that $i$-th subsystem is characterized at time $t \in \mathbb{R}_{+}$only by the parameter $x_{i}(t)$, which is the quantity of unprocessed material within the $i$-th subsystem at time $t$. The state of the whole network is denoted by $x(t)=\left(x_{1}(t), \ldots, x_{n}(t)\right)^{T}$. A 


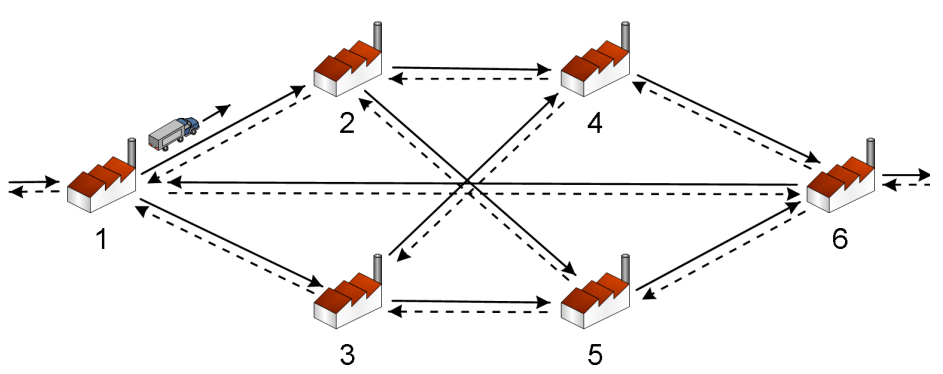

Fig. 1 Example of supply network

subsystem can get material from an external source, which is denoted by $u_{i}$ (external inputs), and from subsystems of the network (internal inputs).

Let the $i$-th subsystem processes the raw material from its inventory with the rate $\tilde{f}_{i i}(t, x(t)) \geq 0$ and sends the produced goods (measured in units of unified material) to the $j$-th subsystem with the rate $\tilde{f}_{j i}(t, x(t))$. Thus, the total rate of the distribution from the $i$-th subsystem to other subsystems is $\sum_{j=1}^{n} \tilde{f}_{j i}(t, x(t))$. It may send a part of production to the customers not considered in the network.

In the version of the model, that we consider in this contribution, we do not distinct the transport of the production out of the network and the losses of production in the manufacture process. However, one can slightly generalize the model, to include the systems with outputs, where output is the total flow of goods out of the system. In this case it is useful to use instead of input-to-state stability (see Section 3) the notion of input-to-output stability.

For general functions $\tilde{f}_{j i}$ it is hard to derive stability conditions. Therefore we consider the following case: $\tilde{f}_{j i}(t, x(t))=c_{j i}(x(t)) \tilde{f}_{i}\left(x_{i}(t)\right), c_{j i}(x) \in \mathbb{R}_{+} \forall x \in \mathbb{R}_{+}^{n}$ and $\tilde{f}_{i i}(t, x(t))=\tilde{c}_{i i}(x(t)) \tilde{f}_{i}\left(x_{i}(t)\right), \tilde{c}_{i i}(x) \in \mathbb{R}_{+}, x \in \mathbb{R}_{+}^{n}$, where $\tilde{f}_{i} \in \mathscr{K}$ is proportional to the processing rate of the system, $c_{j i}(x(t)), i \neq j$ are some positive distribution coefficients and $\tilde{c}_{i i}(x(t)) \geq 0$. We will denote them $c_{j i}(t)$ for the sake of brevity.

Under these assumptions the dynamics of the $i$-th subsystem is described by ordinary differential equations:

$$
\dot{x}_{i}(t)=\sum_{j=1, j \neq i}^{n} c_{i j}(t) \tilde{f}_{j}\left(x_{j}(t)\right)+u_{i}(t)-\tilde{c}_{i i}(t) \tilde{f}_{i}\left(x_{i}(t)\right), i=1, \ldots, n .
$$

Denoting $c_{i i}:=-\tilde{c}_{i i}$ we can rewrite the above equations as an interconnected system in a vector form

$$
\dot{x}(t)=C(t) \tilde{f}(x(t))+u(t),
$$

where $\tilde{f}(x(t))=\left(\tilde{f}_{1}\left(x_{1}(t)\right), \ldots, \tilde{f}_{n}\left(x_{n}(t)\right)\right)^{T}, u(t)=\left(u_{1}(t), \ldots, u_{n}(t)\right)^{T}$ and $C(t) \in$ $\mathbb{R}^{n \times n}$.

The distribution coefficients may be used as controls for some central or autonomous control method. If the elements of the subsystems are controlled au- 
tonomously, then every subsystem can use their own control method, and it is important to have conditions, that guarantee, that the system is stable.

If the system is controlled by central planning method, then we assume, that there exists a planning center, that chooses the distribution coefficients to reach some aim, for example, to maximize the total production rate of the network, to minimize the transportation costs, to improve the stability properties of the system.

In the following we show, how one can use both methods to obtain the stability conditions and gain some information, how to construct the possible distributional coefficients.

\subsection{Optimal strategies}

In this section we model the planning center of a production network (1) as an object, that controls directly all distributional coefficients $c_{i j}, i, j=1, \ldots, n, i \neq j$. That is, we assume in this section, that $c_{i j}$ are functions only of $t$, and do not depend on $x(t)$ explicitly.

For convenience we assume, that the aim of the planing center is to minimize the costs for the storage of the material over the time $[0, T]$, which can be modeled by the following functional:

$$
S_{1}=\int_{0}^{T} \sum_{i=1}^{n} x_{i}^{2}(s) d s \rightarrow \min .
$$

To achieve this goal it uses the information about the inputs $u_{i}, i=1, \ldots, n$ (which is known for some time interval $[0, T]$ ).

The controls, that a planning center may use to achieve this aim, are the distributional coefficients $c_{i j}(t) \geq 0, i, j=1, \ldots, n, i \neq j$. We assume, that there are given the following constraints to the distributional coefficients:

$$
\sum_{i=1, i \neq j}^{n} c_{i j}(t)=k_{j}, j=1, \ldots, n
$$

They tell us, that each node of a network can control only the portions of goods, distributed to other nodes, but cannot control the total distribution rate.

Our aim is to prove, that under made assumptions the functions $c_{i j}, i, j=$ $1, \ldots, n, i \neq j$ have to be, for realistic inputs, piecewise constant functions.

The necessary conditions of extremum are given by the Pontryagin Maximum Principle. The Hamiltonian for system (1) with objective functional (3) is given by:

$$
H=\sum_{i=1}^{n} p_{i}(t)\left(c_{i i}(t) f_{i}\left(x_{i}(t)\right)+\sum_{j=1, j \neq i}^{n} c_{i j}(t) f_{j}\left(x_{j}(t)\right)+u_{i}(t)\right)-\lambda_{0} \sum_{i=1}^{n} x_{i}^{2}(t) .
$$

Here $p_{i}, i=1, \ldots n$ and $\lambda_{0}$ are the Lagrange coefficients. 
According to Pontryagin Maximum Principle (see, e.g., [1]), $c_{i j} \geq 0$ have to be chosen such, that condition (4) holds, and $H \rightarrow$ max.

We cannot control inputs $u_{i}$ and consumption rate $c_{i i}, i=1, \ldots, n$, and therefore maximization of (5) leads us to the following problem:

$$
\begin{gathered}
\sum_{i=1}^{n} p_{i}(t)\left(\sum_{j=1, j \neq i}^{n} c_{i j}(t) f_{j}\left(x_{j}(t)\right)\right)=\sum_{i=1}^{n} \sum_{j=1, j \neq i}^{n} p_{i}(t) c_{i j}(t) f_{j}\left(x_{j}(t)\right)= \\
\sum_{j=1}^{n}\left(\sum_{i=1, i \neq j}^{n} p_{i}(t) c_{i j}(t)\right) f_{j}\left(x_{j}(t)\right) \rightarrow \text { max. }
\end{gathered}
$$

To maximize this linear combination of $f_{j}\left(x_{j}(t)\right)$, which are nonnegative for all $t \geq 0$. The coefficients $c_{i j}$ are dependent only on the distributional coefficients from the same sum in brackets, therefore the terms in brackets may be maximized independently

$$
\forall j=1, \ldots, n \quad \sum_{i=1, i \neq j}^{n} p_{i}(t) c_{i j}(t) \rightarrow \max .
$$

Using constraints, we conclude, in case, if the set $\left\{p_{1}(t), \ldots, p_{n}(t)\right\}$ has the unique maximal element, that

$$
\forall j=1, \ldots, n, \forall s=1, \ldots, n, s \neq j, c_{s j}(t)=\left\{\begin{array}{l}
k_{j}, s=\arg \max \left\{p_{1}(t), \ldots, p_{n}(t)\right\} \\
0, \text { otherwise }
\end{array}\right.
$$

If for some $t$ there exists $I \subset\{1, \ldots, n\}$, such that $\forall i, j \in I$ it holds, that $p_{i}=p_{j}$ and $\forall i \in I, \forall k \in\{1, \ldots, n\} p_{i} \geq p_{k}$. Then

$$
\forall j=1, \ldots, n, \forall s=1, \ldots, n, s \neq j, c_{s j}(t)=\left\{\begin{array}{l}
\in\left[0, k_{j}\right], s \in I \\
0, \text { otherwise }
\end{array}\right.
$$

and it must still hold (4). These controls are called mixed controls. But the situation, that the values of at least two functions $p_{i}, i=1, \ldots, n$ will be identical on some time interval $\left[t_{0}, t_{1}\right], t_{1}>t_{0}$ is quite unrealistic, therefore we see, that the controls $c_{i j}$ are (with exception to quite unrealistic inputs) piecewise constant functions.

One can interpret it, that in every moment one has to send the goods to the node, that "promises" the biggest virtue.

\subsection{Autonomous control methods}

In this subsection we describe several autonomous control policies. They can be implemented in the model of a production network by the terms $c_{i j}(t)$ in (1). These different policies were developed and investigated in the research project CRC637 (see the Acknowledgement). 
The queue length estimator (QLE) policy enables logistic objects in a production network to estimate the waiting, processing and transportation times of different alternative processing resources. A logistic object will decide for the plant with the lowest estimated waiting, processing and transportation time. It uses local information to evaluate the states of the alternatives. It was shown in [26] that the application of this policy leads to a better systems performance regarding throughput times compared to classical scheduling algorithms in highly dynamic situations. In the model (1) the distribution rates of the QLE policy can be defined, for example, by $c_{i j}(t):=c_{i j}^{q}(t)$ and

$$
c_{i j}^{q}(t):=\frac{\frac{1}{x_{i}(t)+\varepsilon}}{\sum_{k} \frac{1}{x_{k}(t)+\varepsilon}},
$$

where the index $k$ denotes all subsystems which get material from subsystem $\mathrm{j}$ and $\varepsilon>0$, arbitrarily small, is inserted to let the fraction be well-defined. The interpretation of $c_{i j}^{q}$ is in simple words the following: if the queue length of the $i$ th subsystem is small, then more material will be sent to subsystem $i$ in contrast to the case where $x_{i}$ is large and $c_{i j}^{q}$ is small.

Similar to the QLE, the due date (DUE) policy estimates waiting, processing and transportation times. While the QLE uses this information for minimizing throughput times of logistic objects, the DUE policy orientates at the tardiness of logistic objects. A logistic object using this policy decides for an alternative resource which offers the lowest difference between estimated due date and pre-planned due date ([23]).

The pheromone based policy is a bio-inspired strategy. The approach is based on the idea to imitate the process of marking possible routes to food sources by ants. Ants emit pheromones between the nest and food sources. Other ants can detect those pheromones and will follow the trail with the highest concentration of pheromones. This is transferred to production networks: During the production process, the logistic objects leave information about their transportation, processing and waiting times at a corresponding machine or plant. Following logistic objects compare this artificial pheromone concentration and choose a production line. Thus, the pheromone concentration depends on transportation, waiting and processing times of previous logistic objects $([4,25])$. To model the evaporation process of natural pheromones a constant $v_{i} \in \mathbb{R}_{+}$is inserted in the definition of the distribution rates, which can be chosen, for example, as

$$
c_{i j}(t)=c_{i j}^{p}(t):=\left(1-v_{i}\right) \frac{\tilde{f}_{i}\left(x_{i}(t)\right)}{\sum_{k} \tilde{f}_{k}\left(x_{k}(t)\right)+\varepsilon}+\sum_{k \neq i} v_{k} \frac{\tilde{f}_{k}\left(x_{k}(t)\right)}{\sum_{q} \tilde{f}_{q}\left(x_{q}(t)\right)+\varepsilon},
$$

where $k, q$ are indices denoting the subsystems which get material from subsystem $\mathrm{j}, \varepsilon>0$ and $0 \leq v_{i} \leq 1$ is the evaporation constant of the $i$ th subsystem. By the evaporation constant $v_{i}$ one can justify the PHE method in order to increase the performance or robustness of the network. 
The honey bee algorithm (HBA) is another bio-inspired strategy. It uses the foraging mechanisms of honey bees' colonies. In nature, bees advertise possible food sources with a so called 'waggle dance'. The duration of this dance depends on the ratio between energy consumption of the flight (between hive and food source) and available energy of the source. The probability of bees recognizing the dance of a dancing bee is proportional to the dancing duration. According to this principle logistic objects are able to advertise different alternative production resources by means of the machining quality, which is determined by calculation of the ratio of value added and the throughput time needed for this step ([24]).

The natural process, which inspires the chemotaxis (CHE) policy, differs from the PHE and the HBA policy. It is not inspired by coordination principals of social insects, but on movement processes coming from microbiology. Natural bacteria are able to direct their movement according to the concentration of attractants (e.g., food substances) or repellants (e.g., toxic substances). Therefore, bacteria perform a random biased walk to find appropriate food sources. This basic movement principle is transferred to autonomous decision making by the CHE policy. Logistic objects using this policy decide according to the gradient of logistic target values of different decision alternatives ([22]).

In the following section we introduce a couple of parameters that characterize the information distribution throughout the network.

\subsection{Properties of a network}

As we will see, neither autonomous control nor central planning is a panacea for solving of all problems, that arise in the control of production networks.

The central planning, if it is theoretically and practically possible, may provide better results, than autonomous control. But usually the problems, that arise in real networks are so complex, that the decisions, made by the central planning method will take a lot of resources (e.g., too much computation time), and the state of the systems changes so quickly, that the obtained results will have in a new situation only limited applicability.

Another problem, that arises in supply networks, is the lack of information in the network, when it is sent from the subsystems of the network to the planning center. Managers, that make decisions for the subsystems have always more information about their own systems, than they send to the planning center. Consequently, although the planning center has information about all the nodes of the network, this information is not exact, and decisions of a planning center may be not accurate enough or at all harmful for the system.

In this section we introduce three properties, that allow to choose the appropriate method to control a production network:

- $n$ - Size of a network.

- $p$ - A number, that characterizes availability of information about the network.

- $L$ - Loss of information in hierarchical structure of a network. 
In networks of small size the central control can provide better results, because the complexity of the problem is lower than for large scale networks, and central control can use the knowledge of the state of all the system more effectively.

Availability of information in a production network plays also a big role. The more knowledge has the planning center, the more effective will be the work of control center, and the less information is given, the better results will be provided by the autonomous control strategy.

We collect these considerations to the following table:

\begin{tabular}{|c|c|c|}
\hline & $n$ is small & $n$ is large \\
\hline$p$ is small & Both & Autonomous control \\
\hline$p$ is large & Central planning & Mixed strategy \\
\hline
\end{tabular}

With the increase of $L$, the advantages of the autonomous control over central planning also increase.

For modeling of autonomous control it is important to investigate the stability of the network. We have to be sure, that the decisions, made by subsystems without (or almost without) communication with each other, cannot induce the unstable behavior of the system.

Since stability is important for the performance and vitality of the network we provide a framework, that may be used to gain some information about stability of the network, and we state the recent results about input-to-state stability of the model of production network, shown in the Section 2.1.

\section{Stability of production networks}

In this section we recall stability properties and tools how to check whether a system is stable.

One possibility to model production networks are ordinary differential equations (ODEs). An ODE is of the form

$$
\dot{x}(t)=f(x(t), u(t)), t \in \mathbb{R}_{+},
$$

where $x \in \mathbb{R}^{N}$ denotes the state of the system, $u \in \mathbb{R}^{M}$ is the essentially bounded measurable external input and $f: \mathbb{R}^{N} \times \mathbb{R}^{M} \rightarrow \mathbb{R}^{N}$ describes the system dynamics. ODEs describe the evolution of the state of the system with continuous time $t \in \mathbb{R}_{+}$, where $\mathbb{R}_{+}:=[0, \infty)$.

To have existence and uniqueness of a solution of a system of the form (6) the function $f$ is assumed to be a locally Lipschitz continuous function. The solution is denoted by $x\left(t ; x_{0}, u\right)$ or $x(t)$ for short, where $x_{0}:=x(0)$ is the initial condition.

In general, production networks consist of $n \in \mathbb{N}$ interconnected systems of the form

$$
\dot{x}_{i}(t)=f_{i}\left(x_{1}(t), \ldots, x_{n}(t), u_{i}(t)\right), t \in \mathbb{R}_{+}, i=1, \ldots, n,
$$


where $x_{i} \in \mathbb{R}^{N_{i}}, u_{i} \in \mathbb{R}^{M_{i}}$ and $f_{i}: \mathbb{R}^{\sum_{j=1}^{n} N_{j}+M_{i}} \rightarrow \mathbb{R}^{N_{i}}$ are locally Lipschitz continuous functions. Here, $x_{j}, j \neq i$ can be interpreted as internal inputs of the $i$-th subsystem and the solution is denoted by $x_{i}\left(t ; x_{i}^{0}, x_{j}, j \neq i, u_{i}\right)$ or $x_{i}(t)$ for short, where $x_{i}^{0}:=$ $x_{i}(0)$ is the initial condition.

If we define $N:=\sum_{i=1}^{n} N_{i}, M:=\sum_{i=1}^{n} M_{i}, x:=\left(x_{1}^{T}, \ldots, x_{n}^{T}\right)^{T}, u:=\left(u_{1}^{T}, \ldots, u_{n}^{T}\right)^{T}$ and $f=\left(f_{1}^{T}, \ldots, f_{n}^{T}\right)^{T}$, then the interconnected system of the form (7) can be written as one single system of the form (6), which we call the whole system.

The purpose of this section is to analyse production networks, which can be written in the form (7), in view of stability. For this purpose we introduce:

Definition 1. We define following classes of functions:

$$
\begin{aligned}
\mathscr{P} & :=\left\{f: \mathbb{R}^{n} \rightarrow \mathbb{R}_{+} \mid f(0)=0, f(x)>0, x \neq 0\right\} \\
\mathscr{K} & :=\left\{\gamma: \mathbb{R}_{+} \rightarrow \mathbb{R}_{+} \mid \gamma \text { is continuous, } \gamma(0)=0 \text { and strictly increasing }\right\} \\
\mathscr{K}_{\infty} & :=\{\gamma \in \mathscr{K} \mid \gamma \text { is unbounded }\} \\
\mathscr{L} & :=\left\{\gamma: \mathbb{R}_{+} \rightarrow \mathbb{R}_{+} \mid \gamma \text { is continuous and strictly decreasing with } \lim _{t \rightarrow \infty} \gamma(t)=0\right\} \\
\mathscr{K} \mathscr{L} & :=\left\{\beta: \mathbb{R}_{+} \times \mathbb{R}_{+} \rightarrow \mathbb{R}_{+} \mid \beta \text { is continuous, } \beta(\cdot, t) \in \mathscr{K}, \beta(r, \cdot) \in \mathscr{L}, \forall t, r \geq 0\right\}
\end{aligned}
$$

We call functions of class $\mathscr{P}$ positive definite.

Definition 2. 1. System (6) is locally input-to-state stable (LISS) if there exist constants $\rho, \rho_{u}>0, \gamma \in \mathscr{K}$ and $\beta \in \mathscr{K} \mathscr{L}$ such that for all initial values $\left|x_{0}\right| \leq \rho$ and all inputs $\|u\|_{\infty} \leq \rho_{u}$ the inequality

$$
|x(t)| \leq \max \left\{\beta\left(\left|x_{0}\right|, t\right), \gamma\left(\|u\|_{\infty}\right)\right\}
$$

is satisfied $\forall t \in \mathbb{R}_{+}$, where $|\cdot|$ denotes the Euclidean norm and $\|u\|_{\infty}:=$ ess sup $|u(t)|$ is the essential supremum norm. $\gamma$ is called (nonlinear) gain.

2. The $i$-th subsystem of (7) is called LISS if there exist constants $\rho_{i}, \rho_{i j}, \rho_{i}^{u}>$ $0, \gamma_{i j}, \gamma_{i} \in \mathscr{K}$ and $\beta_{i} \in \mathscr{K} \mathscr{L}$ such that for all initial values $\left|x_{i}^{0}\right| \leq \rho_{i}$ and all inputs $\left\|x_{j}\right\|_{\infty} \leq \rho_{i j},\left\|u_{i}\right\|_{\infty} \leq \rho_{i}^{u}$ the inequality

$$
\left|x_{i}(t)\right| \leq \max \left\{\beta_{i}\left(\left|x_{i}^{0}\right|, t\right), \max _{j \neq i} \gamma_{i j}\left(\left\|x_{j}\right\|_{\infty}\right), \gamma_{i}\left(\left\|u_{i}\right\|_{\infty}\right)\right\}
$$

is satisfied $\forall t \in \mathbb{R}_{+} . \gamma_{i j}$ and $\gamma_{i}$ are called (nonlinear) gains.

Note that, if $\rho, \rho_{u}=\infty$ then the system (6) is called (global) ISS and if $\rho_{i}, \rho_{i j}, \rho_{i}^{u}=\infty$ then the $i$-th subsystem of (7) is called (global) ISS. In particular LISS and ISS guarantee that the norm of the trajectories of each subsystem is bounded.

An important tool to verify LISS and ISS, respectively, of a system of the form (7) are Lyapunov functions.

Definition 3. We assume that for each subsystem of the interconnected system (6) there exists a function $V_{i}: \mathbb{R}^{N_{i}} \rightarrow \mathbb{R}_{+}$, which is locally Lipschitz continuous, proper 
and positive definite. Then, for $i=1, \ldots, n$ the function $V_{i}$ is called a LISS Lyapunov function of the $i$-th subsystem of (7) if $V_{i}$ satisfies the following two conditions:

There exist functions $\psi_{1 i}, \psi_{2 i} \in \mathscr{K}_{\infty}$, where $\mathscr{K}_{\infty}$ is the subset of $\mathscr{K}$-functions that are unbounded, such that

$$
\psi_{1 i}\left(\left|x_{i}\right|\right) \leq V_{i}\left(x_{i}\right) \leq \psi_{2 i}\left(\left|x_{i}\right|\right), \forall x_{i} \in \mathbb{R}^{N_{i}}
$$

and there exist $\gamma_{i j}, \gamma_{i} \in \mathscr{K}$, a positive definite function $\mu_{i}$, which is continuous, $\mu_{i}(0)=0$ and $\mu_{i}(r)>0, \forall r \in \mathbb{R}$, and constants $\rho_{i}, \rho_{i j}, \rho_{i}^{u}>0$ such that

$$
V_{i}\left(x_{i}\right) \geq \max \left\{\max _{j \neq i} \gamma_{i j}\left(V_{j}\left(x_{j}\right)\right), \gamma_{i}\left(\left|u_{i}\right|\right)\right\} \Rightarrow \nabla V_{i}\left(x_{i}\right) \cdot f_{i}(x, u) \leq-\mu_{i}\left(V_{i}\left(x_{i}\right)\right)
$$

for almost all $x_{i} \in \mathbb{R}^{N_{i}},\left|x_{i}^{0}\right| \leq \rho_{i},\left|x_{j}\right| \leq \rho_{i j}, u_{i} \in \mathbb{R}^{M_{i}},\left|u_{i}\right| \leq \rho_{i}^{u}, \chi_{i i}=0$, where $\nabla$ denotes the gradient of the function $V_{i}$. Functions $\gamma_{i j}$ are called LISS Lyapunov gains.

Note that, if $\rho_{i}, \rho_{i j}, \rho_{i}^{u}=\infty$ then the LISS Lyapunov function of the $i$-th subsystem becomes an ISS Lyapunov function of the $i$-th subsystem (see [14]). In general the LISS Lyapunov gains are different from the gains in Definition 2.

Condition (8) implies that $V_{i}$ is proper, positive definite and radially unbounded. $V_{i}$ can be interpreted as the energy of a system and the second condition (9) of a Lyapunov function means that if $V_{i}\left(x_{i}\right) \geq \max \left\{\max _{j \neq i} \gamma_{i j}\left(V_{j}\left(x_{j}\right)\right), \gamma_{i}\left(\left|u_{i}\right|\right)\right\}$ holds, then the energy decreases. If $V_{i}\left(x_{i}\right)<\max \left\{\max _{j \neq i} \gamma_{i j}\left(V_{j}\left(x_{j}\right)\right), \gamma_{i}\left(\left|u_{i}\right|\right)\right\}$ then the energy of the system is bounded by the expression on the left side of the previous inequality. Overall, the trajectory of the system is bounded.

Furthermore we define the gain-matrix $\Gamma:=\left(\gamma_{i j}\right)_{n \times n}, i, j=1, \ldots, n, \gamma_{i i}=0$, which defines the map $\Gamma: \mathbb{R}_{+}^{n} \rightarrow \mathbb{R}_{+}^{n}$ by

$$
\Gamma(s):=\left(\max _{j} \gamma_{1 j}\left(s_{j}\right), \ldots, \max _{j} \gamma_{n j}\left(s_{j}\right)\right)^{T}, s \in \mathbb{R}_{+}^{n} .
$$

Note that the matrix $\Gamma$ describes in particular the interconnection structure of the network, moreover it contains the information about the mutual influence between the subsystems, which can be used to verify the (L)ISS property of networks.

Definition 4. $\Gamma$ satisfies the local small gain condition $(L S G C)$ on $\left[0, w^{*}\right]$, provided that

$$
\Gamma\left(w^{*}\right)<w^{*} \text { and } \Gamma(s) \nsupseteq s, \forall s \in\left[0, w^{*}\right], s \neq 0 .
$$

Notation $\nsupseteq$ means that there is at least one component $i \in\{1, \ldots, n\}$ such that $\Gamma(s)_{i}<s_{i}$.

To check whether the interconnected system of the form one has to find a LISS Lyapunov function for each subsystem. If there exists a LISS Lyapunov function for each subsystem then it has the LISS property. Furthermore, if the LISS Lyapunov 
gains satisfy the local small-gain condition, then the whole system of the form (6) is LISS, which we recall in the following theorem (see [13]):

Theorem 1. Consider the interconnected system (7), where each subsystem has an LISS Lyapunov function $V_{i}$. If the corresponding gain-matrix $\Gamma$ satisfies the local small-gain condition (11), then there exist constants $\rho, \rho_{u}>0$, such that the whole system of the form (6) is LISS.

In [12] a similar ISS small-gain theorem for general networks was proved, where the small-gain condition is of the form

$$
\Gamma(s) \nsupseteq s, \forall s \in \mathbb{R}_{+}^{n} \backslash\{0\} .
$$

Now we use the stability notions and Lyapunov functions for a stability analysis of production networks, modeled in Section 2.1 in the form (1) or (2). With help of Theorem 1 we can derive conditions, which guarantee stability of the production network:

Proposition 1. Consider a network as in (2).

1. Assume that the $c_{i j}$ are bounded for all $i, j=1, \ldots, n, i \neq j$ and $\tilde{f}_{j} \in \mathscr{K}_{\infty}, j=$ $1, \ldots, n$. If $\exists a \in \mathbb{R}^{n}, \varepsilon \in \mathbb{R}^{n}, a_{i}>0, \varepsilon_{i}<0, i=1, \ldots, n$ such that the condition $C(t) a<\varepsilon$ holds $\forall t>0$, then the whole network is ISS.

2. Let $\tilde{f}_{j} \in \mathscr{K} \backslash \mathscr{K}_{\infty}$, and $\alpha_{j}:=\sup _{x_{j} \in \mathbb{R}}\left\{\tilde{f}_{j}\left(x_{j}\right)\right\}, j=1, \ldots n, \alpha:=\left(\alpha_{1}, \ldots, \alpha_{n}\right)^{T}$. If $\exists u \in L_{\infty}\left(\mathbb{R}_{+}, \mathbb{R}_{+}^{n}\right)$ and $\exists \varepsilon \in \mathbb{R}^{n}, \varepsilon_{i}<0, i=1, \ldots, n$ such that

$$
C(t) \alpha+\|u\|_{\infty}<\varepsilon,
$$

where $\|u\|_{\infty}:=\left(\left\|u_{1}\right\|_{\infty}, \ldots,\left\|u_{n}\right\|_{\infty}\right)^{T}$, then the whole network (2) is LISS. Furthermore, the constants $\rho$ and $\rho_{u}$ from the Definition 2 may be chosen as $\rho:=\infty$, $\rho_{u}:=\min _{i=1, \ldots, n}\left\|u_{i}\right\|_{\infty}$ and (13) holds for all $w \in L_{\infty}\left(\mathbb{R}_{+}, \mathbb{R}_{+}^{n}\right):\|w\|_{\infty} \leq\|u\|_{\infty}$.

The proof can be found in [6].

For certain scenarios of production networks it may happen that the conditions to guarantee stability in Proposition 1 are conservative, which means that one can find parameters which do not satisfy the conditions, but the trajectory of the state of the network is bounded (stable). The reason is, that the presented stability analysis is a "worst-case" approach.

To refine the conditions in Proposition 1 and to identify parameters which guarantee stability of a certain scenario of a production network a dual approach of the stability analysis and simulations was presented in [17]: At first, one derives parameters constellations which guarantee stability by the help of Proposition 1. A large set of parameter constellations is checked except the few constellations for which the conditions in Proposition 1 are not satisfied. This set of (stable) parameter constellations is refined by simulation runs of the network, where only the parameter constellations are simulated which do not satisfy the conditions in Proposition 1.

In contrast to a pure simulation approach, where the time needed for the identification of parameter constellations which guarantee stability of the network increases 
exponentially by an increasing number of entities or parts within the network, this dual proceeding identifies parameter constellations in an acceptable time. This procedure was shown and explained in more detail in [17] and can be summarized in the following figure:

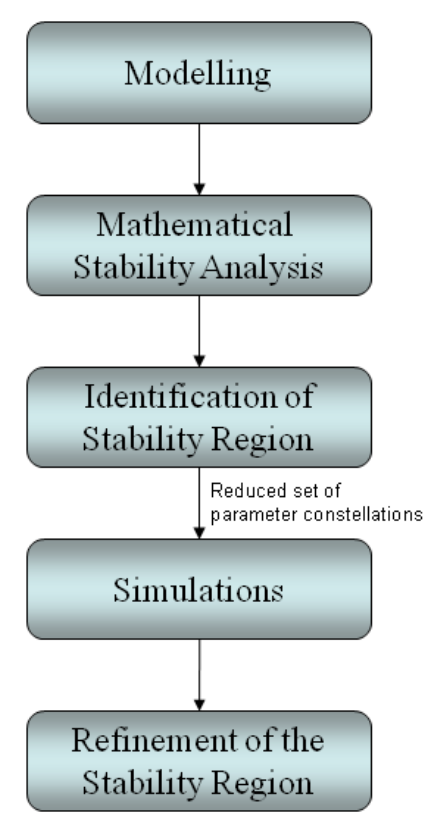

Fig. 2 Scheme of the identification of (stable) parameter constellations

\subsection{Possible generalizations of the model}

Naturally, in production networks the time needed for the transportation of material from one plant to another has to be taken into account. To model time-delays (e.g., transportation times) we use functional differential equations of the form

$$
\dot{x}(t)=f\left(x_{t}, u(t)\right), t>0, x(\tau)=\xi_{0}(\tau), \tau \in[-\theta, 0],
$$

where $t \in \mathbb{R}_{+}, x(t) \in \mathbb{R}^{N}, u(t) \in \mathbb{R}^{M}$ is an essentially bounded measurable input and the function $x_{t}:[-\theta, 0] \rightarrow \mathbb{R}^{N}$ is given by $x_{t}(\tau):=x(t+\tau), \tau \in[-\theta, 0]$, where $\theta$ is the maximum involved delay. $f: C\left([-\theta, 0] ; \mathbb{R}^{N}\right) \times \mathbb{R}^{M} \rightarrow \mathbb{R}^{N}$ is a locally Lipschitz continuous functional on any bounded set.

Using interconnections of time-delay systems one can model general production networks with transportation times, where (1) or (2) must be adapted accordingly 
(see [6]). Also the ISS property has to be defined for systems of the form (14) and interconnected systems of such type.

To check whether a system of the form (14) has the ISS property, one can use Lyapunov-Razumikhin (LR) functions or Lyapunov-Krasovskii (LK) functionals. It was shown in [11] that if each subsystem of an interconnected system with timedelays admits a LR or a LK function(al) and if the small-gain condition (12) is satisfied, then the whole network possesses the ISS property. A certain scenario of an autonomously controlled production network was investigated using the mentioned tools in $[6,7]$.

Another approach to model production networks is to combine continuous dynamics, described by ODEs or functional differential equations, and discrete dynamics. Such systems are called impulsive or hybrid systems and cover, for example, the load and unload of the inventory of a plant. Also time-delays can be considered. The ISS property and the Lyapunov tools have to be defined accordingly. Then, it was shown in [8,9] that a network of impulsive or hybrid systems possesses the ISS property, if there exists a Lyapunov function for each subsystem and the small-gain condition is satisfied.

To include into the model not only transportation times, but the description of the whole transportation process, we may use the partial differential equations (PDE). The simplest model of this art is a standard inhomogeneous transport equation, that takes into account the transportation time and the losses (or increasing) of goods during the transportation. In paper [5] the transport equation was generalized to include the restriction for the traffic flow capacity (in particular, it makes possible to analyze the traffic jams). The input-to-state stability methods for systems, based on partial differential equations, at the time are not developed to the same extent, as the impulsive or time-delay systems. Some preliminary results for certain classes of PDEs were obtained in [10].

To model the central control methods for the logistic networks, one can use the corresponding methods from optimal control of systems with time-delays, systems, based on partial differential equations (see, e.g., [27]) or other classes of systems.

\section{Limitations of Autonomous Control}

To find, what are the limitations of autonomous control, we have to answer firstly the question, what are the limitations, common for both central and autonomous control.

We distinct:

- "essential" limitations, which provide us with bounds, within which the control method may be applied, both theoretically and in practice.

- limitations of effectiveness, which provide us with bounds, within which the method works better, than the other ones. 
Returning to parameters of the networks, introduced in Section 2.4, we can say, that we can identify the following limitations of autonomous control:

Structural limitations: the limitations, that are essential for a system, and do not depend on the type of control strategy. In our model such limitations are the stability bounds of the system. It is clear, that if the processing rate of materials is bounded for all $x_{i}(t)$, then we can always find such an input, that the system will be unstable for all possible controls $c_{i j}$. The condition (13) provides us with a priori stability bounds, within which the stability is guaranteed.

Limitations of information availability: the less information about the network is available, the less effective is the central control. For example, in retail trade there is no exact information about the quantity of customers, the time of purchases etc., therefore the "pure" central planning, in particular, the direct control of all transportations is not effective, if at all possible. Conversely, for the companies, that have strict arrangements with other enterprises (in terms of our model, the inputs are known), the central planning is more effective, and we have here a limitation of effectiveness for autonomous control methods.

In section 2.2 we have shown, that the optimal controls are the piecewise constant functions, consequently, the computation of the optimal control, if values of inputs $u_{i}, i=1, \ldots, n$ are available on time span $[0, T]$, is resolved to the computation of the points, where the controls are discontinuous, what essentially decreases the computation time, needed for a finding of an optimal control. This shows, that for the systems, for which an extensive information is available, the central control has advantages over autonomous control. As long-term strategy (when information about $u_{i}$ increases with time), the model predictive control (see, e.g., [2]) can be used. But if the information is not available, the computation of optimal controls is not possible, and we have to exploit autonomous control strategies.

Of course, at the same time different types of limitations may become important. For example, in the large-scale networks with high availability of information about subsystems we have, that the central planning may become impossible because of the large size of a network (essential limitation of central control), but also an autonomous control has its limitations of effectiveness, because the possibility to use the exact information from all nodes is disregarded.

In this case different mixed strategies may be applied to find the most effective combination of central planning and autonomous control. For example, the results of Section 2.2 can be used to design the autonomous control method.

\section{Conclusions}

We have considered two methods of control of production networks: autonomous and central control. We have shown, that if information about the flows of goods to the system from the outside is known, then the central control has certain advantages in comparison with autonomous control. In particular, in the Section 2.2 we have 
shown, that (in some sense) optimal distributional coefficients have to be piecewise constant functions.

From the other side, if the information about the flows of goods is not available, the central methods loss their effectiveness, and the autonomous control come to a foreground.

We have also outlined the recent research of stability of logistic networks, based on the notion of input-to-state stability, and discussed perspectives of future investigations in this field. In particular, the theorems were provided, that give sufficient conditions for stability of the network (2) in terms of distributional coefficients, that characterize the material flows between the nodes of a network.

The limitations of both central and autonomous control have been discussed. We argue, that usually in real systems the mixed strategy should be used, depending on the properties of the systems, some of which have been considered in this paper.

\section{Acknowledgement}

Sergey Dashkovskiy, Andrii Mironchenko and Lars Naujok are funded by the German Research Foundation (DFG) as part of the Collaborative Research Centre 637 "Autonomous Cooperating Logistic Processes: A Paradigm Shift and its Limitations".

\section{References}

[1] V. M. Alekseev, V. M. Tikhomirov, and S. V. Fomin. Optimal control. Contemporary Soviet Mathematics. Consultants Bureau, New York, 1987. Translated from the Russian by V. M. Volosov.

[2] Frank Allgöwer and Alex Zheng, editors. Nonlinear model predictive control, volume 26 of Progress in Systems and Control Theory. Birkhäuser Verlag, Basel, 2000. Papers from the workshop held in Ascona, June 2-6, 1998.

[3] E. Alvarez. Multi-plant production scheduling in SMEs. Robotics and Computer-Integrated Manufacturing, 23(6):608-613, 2007.

[4] D. Armbruster, C. de Beer, M. Freitag, T. Jagalski, and Ch. Ringhofer. Autonomous Control of Production Networks Using a Pheromone Approach. Physica A, 363(1):104-114, 2006.

[5] D. Armbruster, P. Degond, and C. Ringhofer. A model for the dynamics of large queuing networks and supply chains. SIAM Journal on Applied Mathematics, 66(3):896-920, 2006.

[6] S. Dashkovskiy, M. Görges, M. Kosmykov, A. Mironchenko, and L. Naujok. Modelling and stability analysis of autonomous controlled production networks. 2010. To appear in Logistics Research. 
[7] S. Dashkovskiy, H. R. Karimi, M. Kosmykov, A. Mironchenko, and L. Naujok. Application of the LISS Lyapunov-Krasovskii small-gain theorem to autonomously controlled production networks with time-delays. In Proceedings of SysTol, Nice, France, Oct. 06-08, 2010, pages 765-770, 2010.

[8] S. Dashkovskiy and M. Kosmykov. Stability of networks of hybrid ISS systems. In Proc. of CDC/CCC 2009, The Joint 48th IEEE Conference on Decision and Control and 28th Chinese Control Conference, pages 3870-3875, Shanghai, P.R. China, December 2009.

[9] S. Dashkovskiy, M. Kosmykov, and L. Naujok. Stability of interconnected impulsive systems with and without time-delays using Lyapunov methods, 2010. http://arxiv.org/abs/1011.2865.

[10] S. Dashkovskiy and A. Mironchenko. On the uniform input-to-state stability of reaction-diffusion systems. In Proceedings of the 49th IEEE Conference on Decision and Control, Atlanta, Georgia, USA, Dec. 15-17, 2010, pages 65476552, 2010.

[11] Sergey Dashkovskiy and Lars Naujok. Lyapunov-Razumikhin and LyapunovKrasovskii theorems for interconnected ISS time-delay systems. In Proceedings of the 19th MTNS 2010, Budapest, Hungary, July 5-9, pages 1179-1184, 2010.

[12] Sergey Dashkovskiy, Björn S. Rüffer, and Fabian R. Wirth. An ISS small gain theorem for general networks. Math. Control Signals Systems, 19(2):93-122, 2007.

[13] Sergey N. Dashkovskiy and Björn S. Rüffer. Local ISS of large-scale interconnections and estimates for stability regions. Systems \& Control Letters, 59(3-4):241 - 247, 2010.

[14] Sergey N. Dashkovskiy, Björn S. Rüffer, and Fabian R. Wirth. Small Gain Theorems for Large Scale Systems and Construction of ISS Lyapunov Functions. SIAM Journal on Control and Optimization, 48(6):4089-4118, 2010.

[15] J.-H. Kim and N. A. Duffie. Backlog Control for a Closed Loop PPC System. Annals of the CIRP, 53(1):357-360, 2004.

[16] J. Sauer. Modeling and solving multi-site scheduling problems. In Planning in Intelligent Systems: Aspects, Motivations and Method, pages 281-299. van Wezel, W. and Jorna, R. and Meystel, A.(Eds.), Wiley, 2006.

[17] B. Scholz-Reiter, S. Dashkovskiy, M. Görges, and L. Naujok. Stability analysis of autonomously controlled production networks. 2010. to appear in International Journal of Production Research.

[18] B. Scholz-Reiter, C. de Beer, M. Freitag, and T. Jagalski. Analysing the Dynamics caused by Autonomously Controlled Logistic Objects. In Proceedings of 2nd International Conference on Changeable, Agile, Reconfigurable and Virtual Production (CARV 2007), pages 273-280. ElMaraghy, H. A. and Zaeh, M. F. (Eds.), Windsor, 2007.

[19] B. Scholz-Reiter, Ch. de Beer, and Th. Jagalski. Selbststeuerung logistischer Prozesse in Produktionsnetzen. Industrie Management, 23(1):19-23, 2007. 
[20] B. Scholz-Reiter, M. Freitag, C. de Beer, and T. Jagalski. Modelling dynamics of autonomous logistic processes: Discrete-event versus continuous approaches. CIRP Annals-Manufacturing Technology, 54(1):413 - 416, 2005.

[21] B. Scholz-Reiter, M. Görges, T. Jagalski, and A. Mehrsai. Modelling and Analysis of Autonomously Controlled Production Networks. In Proceedings of the 13th IFAC Symposium on Information Control Problems in Manufacturing (INCOM 09). Moscow, Russia, pages 850-855, 2009.

[22] B. Scholz-Reiter, M. Görges, T. Jagalski, and L. Naujok. Modelling and analysis of an autonomous control method based on bacterial chemotaxis. In Proceedings of the 43rd CIRP International Conference on Manufacturing Systems (ICMS 2010). Neuer Wissenschaftlicher Verlag, Wien, Austria., pages 699-706, 2010.

[23] B. Scholz-Reiter, M. Görges, and T. Philipp. Autonomously controlled production systems - Influence of autonomous control level on logistic performance. CIRP Annals - Manufacturing Technology, 58(1):395-398, 2009.

[24] B. Scholz-Reiter, T. Jagalski, and J. Bendul. Autonomous control of a shop floor based on bee's foraging behaviour. In Proceedings of the 1st International Conference on Dynamics in logistics. LDIC 2007, pages 415-423. Haasis, H.-D. and Kreowski, H.-J. and Scholz-Reiter, B. (eds.), Springer, Berlin, Heidelberg, 2008.

[25] B. Scholz-Reiter, T. Lensing, M. Görges, and L. Dickmann. Classification of Dynamical Patterns in Autonomously Controlled Logistic Simulations Using Echo State Networks. Harbor, Maritime \& Multimodal Logistics Modelling and Simulation, 2010. In press.

[26] B. Scholz-Reiter, H. Rekersbrink, and M. Görges. Dynamic Flexible Flow Shop Problems - Scheduling Heuristics vs. Autonomous Control. CIRP Annals - Manufacturing Technology, 59(1):465-468, 2010.

[27] Fredi Tröltzsch. Optimal control of partial differential equations, volume 112 of Graduate Studies in Mathematics. American Mathematical Society, Providence, RI, 2010. Theory, methods and applications, Translated from the 2005 German original by Jürgen Sprekels.

[28] H.-P. Wiendahl and S. Lutz. Production in Networks. Annals of the CIRPManufacturing Technology, 51(2):1-14, 2002.

[29] K. Windt. Selbststeuerung intelligenter Objekte in der Logistik. In Selbstorganisation - Ein Denksystem für Natur und Gesellschaft. Vec, M. and Hütt, M. and Freund, A. (Eds.), Köln: Böhlau Verlag, 2006.

[30] K. Windt, F. Böse, and T. Philipp. Criteria and Application of Autonomous Cooperating Logistic Processes. In Proceedings of the 3rd International Conference on Manufacturing Research. Advances in Manufacturing Technology and Management. Gao, J. X. and Baxter, D. I. and Sackett, P. J. (Eds.), 2005. 